\title{
Re-sleeve gastrectomy as revisional bariatric procedure after biliopancreatic diversion with duodenal switch
}

\author{
Philipp C. Nett $^{1}$ (D) Dino Kröll ${ }^{1} \cdot$ Yves Borbély $^{1}$
}

Received: 27 June 2015/Accepted: 22 October 2015/Published online: 7 January 2016

(C) Springer Science+Business Media New York 2016

\begin{abstract}
Background Re-sleeve gastrectomy (re-SG) is a possible option to increase weight loss after biliopancreatic diversion with duodenal switch (BPD-DS). We report the feasibility, efficacy and safety of re-SG in patients presenting with long-term weight regain after BPD-DS.

Methods From October 2010 to December 2013, a total of 17 patients (12 female, 5 male) with a mean age of $42.1 \pm 19.4$ years underwent re-SG, mainly because of weight regain after BPD-DS. Re-SG was performed laparoscopically over a 32 French stomach tube.

Results At the time of BPD-DS, the mean weight and BMI of all patients were $130.1 \pm 17.9 \mathrm{~kg}$ and $46.1 \pm 6.5 \mathrm{~kg} / \mathrm{m}^{2}$, respectively. The mean time interval between BPD-DS and re-SG was $63.1 \pm 20.3$ months. At the time of re-SG, the mean weight and BMI were $115.4 \pm 14.2 \mathrm{~kg}$ and $39.8 \pm 5.3 \mathrm{~kg} / \mathrm{m}^{2}$, and the \%EWL after BPD-DS was $22.9 \pm 17.4 \%$. Three conversions (17.6\%) to open surgery were required. No mortality occurred. One patient (5.9\%) developed a leak within the first week after re-SG that was treated conservatively with an endoluminal stent. The mean follow-up was $37.2 \pm 7.1$ months after re-SG. One- and three-year follow-up showed a mean weight, BMI, and cumulative \%EWL of $96.0 \pm 17.1 \mathrm{~kg}, 33.8 \pm 7.3 \mathrm{~kg} / \mathrm{m}^{2}$, and $53.1 \pm 18.3 \% \quad(17 / 17$ patients; $100 \%)$, and $100.3 \pm 21.1 \mathrm{~kg}, \quad 35.1 \pm 8.3 \mathrm{~kg} / \mathrm{m}^{2}$, and $47.2 \pm 19.7 \%$ (13/17 patients; $76 \%)$ after re-SG, respectively.
\end{abstract}

Philipp C. Nett

philipp.nett@insel.ch

1 Department of Visceral Surgery and Medicine, Inselspital, University Hospital Bern, University Bern, 3010 Bern, Switzerland
Conclusions This study shows that re-SG in patients with weight regain after BPD-DS is a feasible, effective and safe option as a revisional bariatric procedure. However, patients have to be carefully considered for revisional surgery since re-SG is associated with the potential risk of surgical complications.

Keywords Bariatric surgery - Revisional surgery · Weight regain - Sleeve gastrectomy · Biliopancreatic diversion with duodenal switch

Biliopancreatic diversion with duodenal switch (BPD-DS) is a very effective bariatric procedure for sustainable longterm weight loss in the treatment of morbid obesity [1-3]. BPD-DS is known for the highest amount of weight loss among all bariatric procedures [3]. Nevertheless, this operation was less commonly performed within the last 20 years because of its side effects including malabsorption and surgically induced malnutrition [1,4]. BPD-DS relies on gastric restriction by sleeve gastrectomy (SG) combined with the malabsorptive biliopancreatic diversion $[2,3]$. Despite its excellent perspective in terms of weight loss, long-term weight regain can also occur after BPD-DS, especially in super-obese patients $[5,6]$. In these patients, a multidisciplinary approach is necessary to determine the contributive factors for weight regain [7]. The re-evaluation includes a psychologist and a nutritionist that has to rule out any presence of a new mental disorder or dietary behavior [8]. Further examinations should also exclude the appearance of gastroesophageal reflux (GERD) or a possible dilation of the SG [9-11]. The treatment than will depend on the different findings of the evaluations.

In BPD-DS, there are only limited options for revisional surgery to correct long-term weight regain such as changing 
the gastric volume or the lengths of the small bowel channels [12]. Re-SG has, therefore, to be considered in these patients as recent reports demonstrate that SG has the tendency to dilate over time $[11,13-15]$. We postulated that reSG could increase further weight loss after BPD-DS when a dilated SG was present. The aim of this study was to analyze the feasibility, efficacy and safety of re-SG in patients presenting with long-term weight regain after BPD-DS.

\section{Materials and methods}

\section{Patients}

Between October 2010 and December 2013, a total of 17 out of 112 patients $(15.2 \%)$ were identified with sustainable weight regain after BPD-DS. The indication for re-SG was determined after a multidisciplinary re-evaluation of the patient including psychological and nutritional counseling, a gastrographin contrast swallow study and a gastroscopy.

\section{Surgical technique}

The surgical procedure was performed starting with the insertion of a video-guided 12-mm trocar in the left upper quadrant of the abdomen. Four additional trocars were placed under the direct view (two 5-mm and two 12-mm trocars). Re-SG started with a complete adhesiolysis of the greater omentum from the abdominal wall and detaching the left liver lobe from the gastric sleeve. A 32 French stomach tube was inserted by an anesthesiologist to reach the pylorus. The Echelon 60-mm linear stapler (Ethicon Endosurgery, Cincinnati, OH, USA) with green or blue loads as a routine choice was used in all procedures. Staple line buttressing was not used, but hemostatic clips were applied whenever required on the gastric staple lines. The resected stomach was removed in a plastic bag through a left flank trocar incision, and a leakage test with methylene blue was performed. A drain was left in place along the staple line, and a leakage test with methylene blue was performed.

\section{Postoperative management}

Thromboembolic prophylaxis was given using twice $40 \mathrm{mg} / \mathrm{d}$ enoxaparin (Clexane) $6 \mathrm{~h}$ after surgery for 4 weeks. Automatic compression devices were used during the whole operating time and were left in place for the first 24-48 h. The patients received a clear liquid diet for the first $24 \mathrm{~h}$ after the procedure and were discharged after 5 days if no postoperative complications occurred. Dietary restrictions included a concept a strict pureed diet for
4 weeks. Follow-up visits were scheduled at our outpatient bariatric clinic at $1,3,6$, and 12 months after re-SG and every 6 months from that time point forward including a clinical examination and a nutritional blood screening test.

\section{Statistical analysis}

Data were analyzed using Prism 6 (version 6.0d; GraphPad Software). Results are presented as mean values with standard deviation (SD) unless specified otherwise. For categorical variables, we calculated the frequencies of the categories of interest. \%EWL was calculated to the ideal weight set at a BMI of $25 \mathrm{~kg} / \mathrm{m}^{2}$.

\section{Results}

At the time of BPD-DS, mean weight of all patients was $130.1 \pm 17.9 \mathrm{~kg}$ (range 108-176) and BMI $46.1 \pm 6.5 \mathrm{~kg} /$ $\mathrm{m}^{2}$ (range 37.5-53.7) (Figs. 1, 2). Obesity-related comorbidities affected all patients, including arterial hypertension in $12(70.6 \%)$, dyslipidemia in 7 (41.2\%), type 2 diabetes mellitus (DM) in $6(35.3 \%)$, and obstructive sleep apnea syndrome (OSAS) in 5 (29.4\%) patients.

The mean time interval between BPD-DS and re-SG was $63.1 \pm 20.3$ months. At the time of re-SG, mean weight was $115.4 \pm 14.2 \mathrm{~kg}$ (range 96-151), BMI $39.8 \pm 5.3 \mathrm{~kg} /$ $\mathrm{m}^{2}$ (range 33.2-47.9), and \%EWL $22.9 \pm 17.4 \%$ (range 3.9-41.9) (Figs. 1, 2, 3). Obesity-related comorbidities at this time affected 15 of 17 patients (88.2\%), including arterial hypertension in $10(58.9 \%)$, dyslipidemia in 7 $(41.2 \%)$, type $2 \mathrm{DM}$ in $5(29.4 .3 \%)$, and OSAS in 4 $(23.5 \%)$ patients.

The mean operative time for re-SG was $107 \pm 83 \mathrm{~min}$ (range 48-240). Three conversions (17.6\%) to open surgery were required due to severe intraabdominal adhesions.

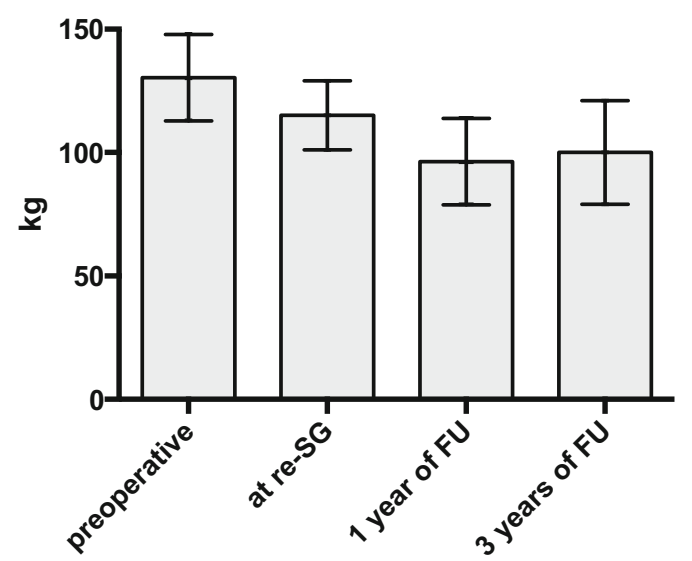

Fig. 1 Comparison of weight $(\mathrm{kg})$ at time of BPD-DS, before re-SG, and during follow-up at 1 and 3 years 


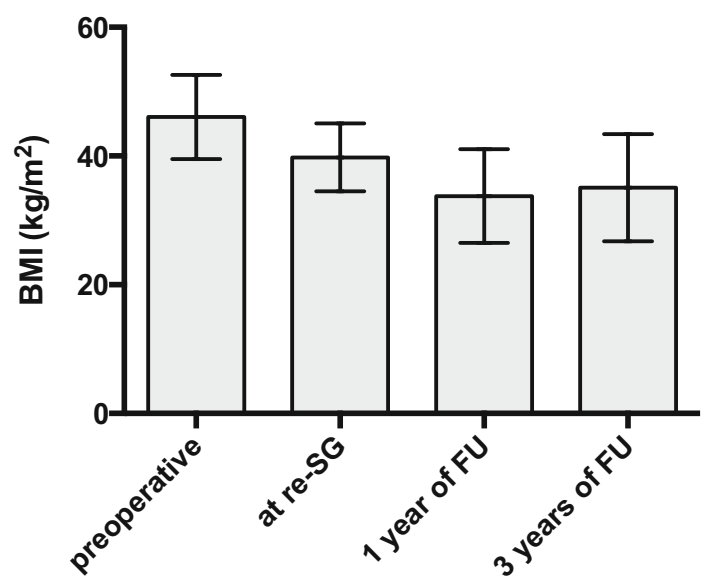

Fig. 2 Comparison of BMI $\left(\mathrm{kg} / \mathrm{m}^{2}\right)$ at time of BPD-DS, before re$\mathrm{SG}$, and during follow-up at 1 and 3 years

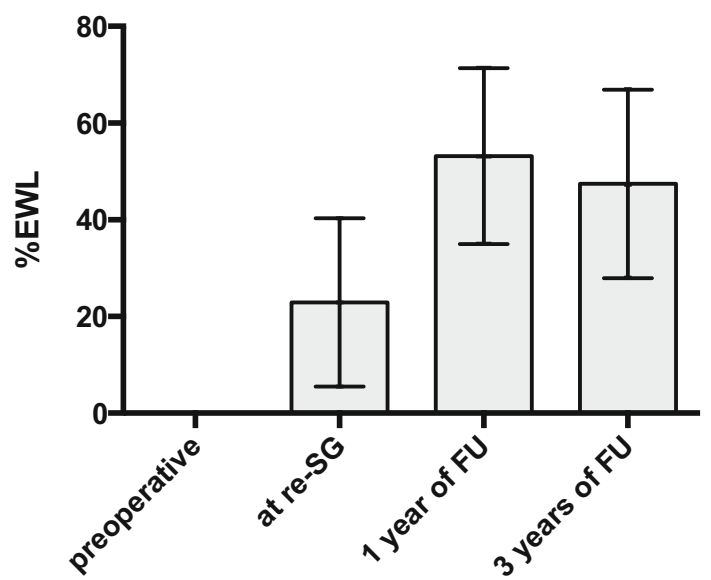

Fig. 3 Comparison of \%EWL at time of re-SG and during follow-up at 1 and 3 years

No mortality occurred. One patient $(5.9 \%)$ presented with a leak at the angle of His within the first week after re-SG that was treated conservatively with an endoluminal stent. The mean hospital stay was $9.3 \pm 19.7$ days (range 5-63).

The mean follow-up was $37.2 \pm 7.1$ months (range 31-48) after re-SG. At 1 year of follow-up (17 patients; complete follow-up), mean weight was $96.0 \pm 17.1 \mathrm{~kg}$ (range 78-135), BMI $33.8 \pm 7.3 \mathrm{~kg} / \mathrm{m}^{2}$ (range 28.7-43.8), and cumulative \%EWL after BPD-DS $53.1 \pm 18.3 \%$ (range 39-132) (Figs. 1, 2, 3). At 3 years of follow-up (13/17 patients; follow-up $76 \%$ ), mean weight was $100.3 \pm$ $21.1 \mathrm{~kg}$ (range $82-138$ ), BMI $35.1 \pm 8.3 \mathrm{~kg} / \mathrm{m}^{2}$ (range 29.5-45.1), and cumulative \%EWL after BPD-DS $47.2 \pm$ $19.7 \%$ (range 35-97) (Figs. 1, 2, 3). Obesity-related comorbidities at this time affected 11 of 17 patients (64.7\%), including arterial hypertension in $7(41.2 \%)$, dyslipidemia in 5 (29.4\%), type $2 \mathrm{DM}$ in $5(29.4 \%)$, and OSAS in $3(17.6 \%)$ patients. There were not more vitamin or mineral deficiencies diagnosed 3 years after secondary reSG compared to 3 years after initial BPD-DS.

During the follow-up, two patients $(11.8 \%)$ required surgery for late complications, including one trocar-site ventral hernia and one incisional ventral hernia repair. Hospitalization was at 3 and 16 months after re-SG with laparoscopic surgery for hernia repair without any complications.

\section{Discussion}

To our knowledge, this is the first study demonstrating that re-SG in patients with weight regain in the long term after BPD-DS is a feasible, effective and safe surgical option to achieve further weight loss after BPD-DS.

BPD-DS is known to achieve the best results in terms of weight loss among all bariatric procedures [1-3]. Nevertheless, weight regain in the long term can also occur after BPD-DS, especially in super-obese patients [5-7]. Marceau et al. [3] reported excellent 20 years results after BPD-DS with a $\% \mathrm{EWL}$ of over $70 \%$, but Biron et al. [16] mentioned that weight regains after BPD-DS is an increasing problem, especially in super-obese patients, doubling the failure rate every 5 years with weight regain. In our series, we identified 17 patients with weight regain at a median follow-up of more than 5 years after BPD-DS. The mean BMI at time of BPD-DS was $46.1 \pm 6.5 \mathrm{~kg} / \mathrm{m}^{2}$, and some of these patients were super-obese (BMI $>50 \mathrm{~kg} / \mathrm{m}^{2}$ ).

In bariatric surgery, weight regain is a common problem [7, 17, 18]. Even after satisfactory weight loss, weight regain can occur if patients go back to bad eating habits and sedentary lifestyle [8, 19]. The reasons for weight regain have to be thoroughly investigated. Evidence has been growing that psychological issues play an essential role [8]. New dietary habits and patient adaptation to the new anatomic circuit are other frequent causes of weight regain [7]. Other reasons may be equally important factors such as excessive alcohol consumption, craving for sweets, fattening foods in general, or dilatation of gastric restriction $[7,8]$. In BPD-DS, weight regain often results in nutritional consequences due to poor intestinal absorption caused by the exclusion of the jejunum from the alimentary tract and the short common channel [12, 20, 21]. Weight regain may represent a return to full energy utilization, but many essential nutrients are still lost, which in BPD-DS may result in severe iron-deficiency anemia, vitamin D deficiency with secondary hyperparathyroidism and chronic malnutrition [20, 21]. This situation results in an obese individual with various nutritional complications that need to be addressed before considering weight loss per se. The follow-up should include medical and psychological guidance, including continuing dietary advice and maintenance of a physical exercise regimen [22]. 
To overcome the problem of weight regain, a multidisciplinary approach is necessary to determine the individuals contributive factors to this problem [7]. Psychological disorders or changes in eating behavior have been correlated with changes in body weight, independent of the reported dietary intake and physical activity [8, 19]. In cases of hyperphagia linked to a dilated gastric sleeve, we offered patients re-SG after BPD-DS. Gagner et al. reported two cases of re-SG in patients with poor weight loss after BPDDS $[14,15]$. When dilation of the SG is present, re-SG appears to be a logical option. In SG, Baltasar et al. [23] reported first on two patients who had undergone re-SG for dilation of SG, with subsequent increased successive weight loss. Other authors reported comparable observation with a substantial of weight regain in up to $23 \%$ of SG $[5,6,11$, 18]. However, consideration should be given to a possible reSG since additional gastric volume restriction might jeopardize the patient's psychological balance [8]. Also, a radiological examination by gastrographin or barium contrast swallow studies does also not seem completely appropriate to evaluate the volume of the SG [11, 24]. During our surgical exploration, we did find sufficient space to place the linear staple next to the stomach tube. After placement of the stomach tube, excessive redundancy was still found toward the antrum and, in some patients, to the fundus.

During a follow-up of more than 3 years after re-SG, we recorded a cumulative \%EWL of $47.2 \%$ after BPD-DS and re-SG compared to \%EWL 22.9 after BPD-DS with weight regain. An average, all patients lost $11 \mathrm{~kg}$ after reSG and showed an improvement of remission of comorbidities in some cases. Although an additional restriction procedure was added to the BPD-DS, not more vitamin or mineral deficiencies were diagnosed 3 years after secondary re-SG compared to 3 years after initial BPD-DS.

In revisional bariatric surgery, the risk of surgical complications is higher and complications might be more difficult to treat $[1,11]$. In the present series, we recorded one $(5.9 \%)$ early complication with a leak at the angle of His within the first week after re-SG. Recent reports have shown that conservative treatment of leakages provides better results than reoperation, with no mortality overall [11]. This was the case in our patient that was treated conservatively with an endoluminal stent. Regarding late complications, we observed two patients $(11.8 \%)$, one with a trocar-site ventral hernia and one with an incisional ventral hernia repair. The latter was one of the three conversions $(17.6 \%)$ which were required for re-SG.

In short, re-SG may be a valid surgical option for patients with weight regain after BPD-DS. Our series show that re-SG after BPD-DS is feasible, effective and safe. However, patients have to be carefully considered for revisional surgery since re-SG is associated with the potential risk of surgical complications.

\section{Compliance with ethical standards}

Disclosures Philipp C. Nett, Dino Kröll, and Yves Borbély have no conflicts of interest.

\section{References}

1. Buchwald H, Oien DM (2013) Metabolic/bariatric surgery worldwide 2011. Obes Surg 23(4):427-436

2. Marceau P, Biron S, Hould FS, Lebel S, Marceau S, Lescelleur O, Biertho L, Simard S (2009) Duodenal switch improved standard biliopancreatic diversion: a retrospective study. Surg Obes Relat Dis 5(1):43-47

3. Marceau P, Biron S, Marceau S, Hould FS, Lebel S, Lescelleur O, Biertho L, Simard S, Kral JG (2015) Long-term metabolic outcomes 5 to 20 years after biliopancreatic diversion. Obes Surg 25(9):1584-1593

4. Buchwald H (2002) Overview of bariatric surgery. J Am Coll Surg 194(3):367-375

5. Dapri G, Cadiere GB, Himpens J (2011) Laparoscopic repeat sleeve gastrectomy versus duodenal switch after isolated sleeve gastrectomy for obesity. Surg Obes Relat Dis 7(1):38-43

6. Dapri G, Cadiere GB, Himpens J (2011) Laparoscopic conversion of Roux-en-Y gastric bypass to distal gastric bypass for weight regain. J Laparoendosc Adv Surg Tech A 21(1):19-23

7. Brethauer SA, Kothari S, Sudan R, Williams B, English WJ, Brengman M, Kurian M, Hutter M, Stegemann L, Kallies K, Nguyen NT, Ponce J, Morton JM (2014) Systematic review on reoperative bariatric surgery: American Society for Metabolic and Bariatric Surgery Revision Task Force. Surg Obes Relat Dis 10(5):952-972

8. Green DD, Engel SG, Mitchell JE (2014) Psychological aspects of bariatric surgery. Curr Opin Psychiatry 27(6):448-452

9. Tutuian R (2014) Effects of bariatric surgery on gastroesophageal reflux. Curr Opin Gastroenterol 30(4):434-438

10. Keidar A, Appelbaum L, Schweiger C, Elazary R, Baltasar A (2010) Dilated upper sleeve can be associated with severe postoperative gastroesophageal dysmotility and reflux. Obes Surg 20(2):140-147

11. Wolnerhanssen B, Peterli R (2014) State of the art: sleeve gastrectomy. Dig Surg 31(1):40-47

12. Topart PA, Becouarn G (2015) Revision and reversal after biliopancreatic diversion for excessive side effects or ineffective weight loss: a review of the current literature on indications and procedures. Surg Obes Relat Dis 11(4):965-972

13. Cesana G, Uccelli M, Ciccarese F, Carrieri D, Castello G, Olmi S (2014) Laparoscopic re-sleeve gastrectomy as a treatment of weight regain after sleeve gastrectomy. World J Gastrointest Surg 6(6):101-106

14. Parikh M, Gagner M (2008) Laparoscopic hiatal hernia repair and repeat sleeve gastrectomy for gastroesophageal reflux disease after duodenal switch. Surg Obes Relat Dis 4(1):73-75

15. Gagner M, Rogula T (2003) Laparoscopic reoperative sleeve gastrectomy for poor weight loss after biliopancreatic diversion with duodenal switch. Obes Surg 13(4):649-654

16. Biron S, Hould FS, Lebel S, Marceau S, Lescelleur O, Simard S, Marceau P (2004) Twenty years of biliopancreatic diversion: what is the goal of the surgery? Obes Surg 14(2):160-164

17. Shimizu H, Annaberdyev S, Motamarry I, Kroh M, Schauer PR, Brethauer SA (2013) Revisional bariatric surgery for unsuccessful weight loss and complications. Obes Surg 23(11): $1766-1773$

18. Noel P, Nedelcu M, Nocca D, Schneck AS, Gugenheim J, Iannelli A, Gagner M (2014) Revised sleeve gastrectomy: another 
option for weight loss failure after sleeve gastrectomy. Surg Endosc 28(4):1096-1102

19. Cambi MP, Marchesini SD, Baretta GA (2015) Post-bariatric surgery weight regain: evaluation of nutritional profile of candidate patients for endoscopic argon plasma coagulation. Arq Bras Cir Dig 28(1):40-43

20. Bal BS, Finelli FC, Shope TR, Koch TR (2012) Nutritional deficiencies after bariatric surgery. Nat Rev Endocrinol 8(9): 544-556

21. Shankar P, Boylan M, Sriram K (2010) Micronutrient deficiencies after bariatric surgery. Nutrition 26(11-12):1031-1037

22. Mechanick JI, Youdim A, Jones DB, Garvey WT, Hurley DL, McMahon MM, Heinberg LJ, Kushner R, Adams TD, Shikora S,
Dixon JB, Brethauer S (2013) Clinical practice guidelines for the perioperative nutritional, metabolic, and nonsurgical support of the bariatric surgery patient-2013 update: cosponsored by American Association of Clinical Endocrinologists, The Obesity Society, and American Society for Metabolic and Bariatric Surgery. Obesity 21(Suppl 1):S1-S27

23. Baltasar A, Serra C, Perez N, Bou R, Bengochea M (2006) Resleeve gastrectomy. Obes Surg 16(11):1535-1538

24. Vidal P, Ramon JM, Busto M, Dominguez-Vega G, Goday A, Pera M, Grande L (2014) Residual gastric volume estimated with a new radiological volumetric model: relationship with weight loss after laparoscopic sleeve gastrectomy. Obes Surg 24(3): 359-363 